\title{
Orden mundial, hegemonía y problemática ambiental
}

\author{
World Order, Hegemony and Environmental Issues
}

Federico di Pasquo, ${ }^{\text {I Gabriela Klier }{ }^{\mathrm{II}} \text { y Tomas Busan }}{ }^{\mathrm{III}}$

\section{Resumen}

El presente trabajo se sostiene a partir de dos ejes centrales. El primero aborda la hegemonía discursiva en torno al tema de la problemática ambiental de orden global, la cual ha "atravesado" la división de los discursos, sean estos científicos, publicitarios, literarios, críticos, o bien provenientes de programas internacionales, de organismos no gubernamentales y de declaraciones ambientales. En el segundo eje, por su parte, se señala que el tema de la problemática ambiental global una vez instalado, ha sido utilizado como justificación para el establecimiento de una gestión de alcance mundial.

Palabras clave: problemática ambiental; discurso social; hegemonía; gestión global; glocalización

\section{Abstract}

This paper is based on two central axes. The first one explores discursive hegemony in global environmental problems, which have cut across the division of scientific, advertising, and literary discourse or that of international programs, nongovernment organizations and environmental statements. The second axis indicates that now that the global environmental problems has been installed, it has been used as a justification for the establishment of global management.

Keywords: environmental issues; social discourse; hegemony; global management; glocalization

\footnotetext{
${ }^{\text {I }}$ Doctorado en Ciencias Biológicas por la Universidad de Buenos Aires, Argentina. Investigador Asistente ConICET en la Facultad de Filosofía y Letras de la Universidad de Buenos Aires, Argentina. Líneas de interés: problemática ambiental, ecología y teoría del conocimiento. Correo electrónico: dipasquof@yahoo.com.ar

II Doctorado en Ciencias Biológicas por la Facultad de Ciencias Exactas y Naturales de la Universidad de Buenos Aires, Argentina. Posdoctorado Conicet en el Centro Científico Tecnológico Patagonia Norte. Líneas de interés: biología de la conservación, complejidad y feminismo. Correo electrónico: gabrielaklier@gmail.com

III Estudiante de Licenciatura en Filosofía en la Universidad de Buenos Aires, Argentina. Líneas de interés: problemática ambiental, ordenamiento territorial y servicios ecosistémicos. Correo electrónico: tomasemiliobusan@gmail.com
} 


\section{Introducción}

La problemática ambiental (de ahora en adelante PA) emergió como tema a tratar durante la décadas de 1960 y 1970 y se puede afirmar que ha tenido un importante alcance hasta el presente (Gudynas, 2015). Sus primeras manifestaciones tuvieron lugar en los países llamados "occidentales" a partir de un conjunto de grupos ambientalistas (los denominados "verdes"). Fue gracias a las protestas de estos grupos que la PA cobró suficiente visibilidad para que luego diferentes instituciones y estamentos sociales abordaran dicha temática, entre ellas se pueden mencionar: empresas multinacionales, ONG, institutos de investigación, universidades, medios de comunicación, entre otros. Estas instituciones y estamentos sociales han generado el "relieve" sobre el cual diferentes discursos dirigidos a la PA circulan y se distribuyen. Es decir, fue a partir de las décadas mencionadas, que se ha ido estableciendo un régimen de circulación de discursos serios sobre la PA.

Algunos de estos discursos fácilmente reconocibles son: el discurso de disciplinas como la ecología (con artículos académicos o libros técnicos sobre manejo de especies), la economía y el derecho (con reglamentos económicos y jurídicos sobre el uso de recursos naturales), el discurso publicitario (con documentales y propaganda), el discurso ambiental (con panfletos de protesta) o también, el discurso de programas internacionales (con informes sobre el manejo de fauna o informes sobre el estado del ambiente). En este trabajo nos proponemos hacer un primer análisis del discurso social vinculado con el tema de la PA. Para abordar este análisis nos concentraremos en el estudio de diferentes discursos que se dieron en el periodo que va del año 1989 a 1995. Dicha periodización encuentra su justificación en que el tema de la PA se instaló masivamente durante este periodo (Delgado, 2002). A la vez dicha etapa funciona como precedente para discusiones más recientes, como aquellas en torno al concepto de glocalización (Meyer, 2000; Roudometof, 2005).

Posiblemente uno de los aspectos más preocupantes de la mencionada PA es que la misma parece comprometer a todo el sistema terrestre. De aquí que la gestión del ambiente se ha presentado, desde mediados del siglo pasado, como uno de los grandes desafíos de las sociedades occidentales. Después de todo, el calentamiento global, la pérdida de biodiversidad, la contaminación de los océanos, la destrucción de la capa de ozono, la lluvia ácida o la acumulación de desechos tóxicos no respetan las fronteras levantadas por los Estados nación. Es en este contexto, que el lema "Pensar globalmente y actuar localmente" se ha forjado y consolidado reemplazando aquel lema inicial de las primeras ONG: "Pensar localmente y actuar globalmente".

Este último implicaba que diferentes grupos locales distribuidos a lo ancho del planeta fueran formando redes dirigidas a fortalecer luchas y reclamos dirigidos al cuidado del ambiente. La transformación de este lema, en contraste, parece sugerir que actualmente los grupos locales deben recibir lineamientos de acción elaborados en otros lugares y además, parece sentar las bases 
para una gestión del globo (Latchinian, 2010). Entonces ¿en qué consiste esta nueva propuesta de primero pensar globalmente para luego actuar localmente? O para ser más precisos, ¿el discurso global asociado a la PA, puede "arrastrar" el riesgo de prácticas geocráticas universales que profundicen las asimetrías entre lo global y lo local?

Con relación a estas preguntas, el objetivo general del presente trabajo es poner en evidencia cómo el tema de la PA puede ser funcional a la propuesta de un orden mundial. Sostenemos que el tema de la PA global ha sido utilizado como justificación para sugerir una administración de alcance mundial. La elaboración de este objetivo se realizó dividiendo el trabajo en cinco apartados. En el segundo presentaremos cierta marca que ha sido asignada a la PA y que puede ser rastreada en diferentes discursos: la marca global. En el tercer apartado, se analizarán siete tipos distintos de discursos, en los cuales se puede reconocer dicha marca. En el apartado cuarto, se argumentará en favor de una hegemonía de lo global en el seno del discurso social de la PA y se presentará cierta aspiración a un orden mundial que se correlaciona con la hegemonía mencionada. Finalmente, ofrecemos algunas consideraciones sobre cómo la PA puede aparecer como justificación para el establecimiento de un orden mundial.

\section{Definiciones y metodología}

Antes de avanzar con el análisis propuesto es importante clarificar algunas categorías conceptuales. Estas categorías, si bien tienen una fuerte deuda con el canadiense Marc Angenot y con el argentino Eliseo Verón, fueron corregidas en función de las fuentes aquí consideradas (Angenot, 2010; Verón, 1974). Precisamos a continuación algunas de estas categorías:

Tema - Por tema debe entenderse aquello de lo que tratan o bien, aquello a lo que remiten los discursos analizados. Como ya hemos mencionado, los discursos aquí considerados estarán dirigidos al tema de la PA. Vale agregar que cada discurso se encuentra enfocado en distintos aspectos de dicho tema.

Sincronía y discurso social - Los discursos aquí analizados pertenecen al periodo que va de 1989 al año de 1995. En este sentido, realizamos arbitrariamente un "corte" sincrónico para intentar dar cuenta de "todo" lo dicho durante ese periodo sobre el tema que nos ocupa, la PA. O dicho con otras palabras, intentamos reconstruir buena parte del discurso social de la PA. Ahora bien, para abordar "todo" lo dicho sobre el tema, apelamos a la perspectiva abierta por Angenot. Este autor caracterizaba el discurso social como "todo lo que se dice y se escribe en un estado de sociedad, todo lo que se imprime, todo lo que se habla públicamente o se representa hoy en los medios electrónicos" (Angenot, 2010: 21). A esta caracterización del discurso social que pretende abarcar "todo" lo dicho, se agrega también que la "sincronía debe admitir el surgimiento de puntos de vin- 
culación" (Angenot, 1998: 72) entre los discursos analizados. Bajo esta perspectiva y retomando el tema que nos ocupa, el corte sincrónico permitirá el estudio del "hilvanado" o bien, de los "puntos de vinculación" que se dan entre los diferentes discursos analizados.

Discurso y condiciones de producción - Habiendo reparado brevemente en la noción de discurso social resulta necesario precisar mejor qué entendemos por discurso. Consideramos al discurso como un conjunto de enunciados que remiten a las mismas condiciones de producción (Foucault, 2008a; Castro, 2011). Estas condiciones de producción pueden venir dadas por una variedad de elementos, tales como sectores sociales, estatus, los "roles" de los hablantes, y la función que tiene el discurso (Foucault, 2013; Maingueneau, 1980; Verón, 2004). En este trabajo nos conformaremos con establecer cuáles son los ámbitos o sectores sociales vinculados con los discursos analizados (como por ejemplo: empresas multinacionales, disciplinas o institutos de investigación). A este respecto Verón (1996: 13) menciona:

Dado que una teoría de los discursos sociales parte del supuesto de que las unidades de análisis significativas, en lo que hace al discurso, deben estar asociadas a condiciones sociales de producción más o menos estables, parece lógico situarse dentro del marco de contextos institucionales fácilmente identificables.

En relación con lo antes dicho y retomando esta última apreciación de Verón, podemos establecer una tipología de los discursos dirigidos a la PA, entre los que destacamos: el discurso ambiental, el de las organizaciones no gubernamentales (ONG), el discurso de disciplinas, el discurso de la publicidad "verde", el de la literatura ambiental, el discurso de las declaraciones ambientales y el de programas internacionales. La tipología recién presentada, nos permitió identificar a cada discurso con (al menos) un ámbito o sector social determinado, poniendo sobre relieve que dichos discursos difieren en sus condiciones de producción.

El método comparativo y los invariantes discursivos - Resulta pertinente destacar la estrategia metodológica que nos permitirá estudiar los discursos aquí considerados. A este respecto, el método consistió en una comparación entre los distintos discursos dirigidos al tema de la PA. A ello se agregaron dos especificaciones: la primera es que los discursos comparados suponen condiciones de producción diferentes, y la segunda especificación, es que de dicha comparación se espera obtener aquello que permanece invariante a través de estos discursos. En este sentido, Verón (1987: 138) menciona:

Se ve con claridad que la razón por la cual jamás se puede analizar un discurso "en si mismo" es [...] porque, tratándose de una búsqueda de invariantes discursivos, asociados a determinadas condiciones de 
producción, la única manera de hacer visibles estos invariantes es hacer variar las condiciones: jamás se puede trabajar sobre un discurso; hay que comparar siempre discursos sujetos a condiciones productivas diferentes.

Bajo esta perspectiva metodológica, esperamos entonces poder indicar en qué son equivalentes los discursos comparados, es decir, qué "unidad" o "fragmento discursivo" propuesto permanece invariante (Verón, 2004).

La marca global de la PA - Una sola característica del tema de la PA nos interesa destacar para los fines del trabajo: la PA ha sido frecuentemente asociada a dimensiones planetarias. Dicho con otras palabras, el tema de la PA ha descansado sobre grandes regiones geográficas o si se quiere, sobre dimensiones espaciales de magnitudes amplias (di Pasquo et al., 2011; di Pasquo, 2013; di Pasquo, 2015). Al respecto Lynton Keith Caldwell (1993: 140), en su libro Ecología. Ciencia y política medioambiental, menciona: "La adopción de una perspectiva planetaria ha permitido a los seres humanos ver el mundo dentro del contexto de toda la Tierra". En el mismo libro agrega: "En el discurso corriente, 'mundial' y 'global' indican dimensiones planetarias" (Caldwell, 1993: 139). En relación con estas citas, puede señalarse que los términos "Tierra" o "Mundo" cumplen una función equivalente al indicar dimensiones planetarias. Y de igual modo, se asume esta dimensión global cuando se menciona la vida sobre la Tierra; por ejemplo, cuando se hace referencia a la "Biósfera". Para los fines de este trabajo interesa destacar que todas éstas fueron (y son) maneras de referir una dimensión planetaria. ${ }^{1}$

Reconocida esta dimensión global del tema de la PA, podemos volver sobre la noción de invariante discursivo antes presentada. En esta dirección, consideraremos como "unidad" invariante a esta marca global, la cual se podrá rastrear en ciertos enunciados de longitud variable. O bien se podrá rastrear en cualquiera de sus posibles variaciones: “mundial”, "Mundo", "Tierra”, "Biósfera”, entre otros.

\section{Comparación: dominancia interdiscursiva sobre la PA}

En este apartado nos concentraremos en comparar y presentar mediante una tabla, un conjunto de discursos dirigidos al tema de la PA. Como ya hemos adelantado, entre los discursos indagados se encontrarán:

\footnotetext{
${ }^{1}$ Es importante destacar que los problemas ambientales descansan en una variedad de dimensiones espacio-temporales: escala local, escala de paisaje, escala regional, escala continental y escala global. Estas dimensiones espacio-temporales inherentes a la ecología disciplinar (rama de la biología) encuentran un correlato parcial en ciertos niveles de intervención de la PA: nivel local, nivel regional, nivel nacional y nivel internacional. Con todo, aquí focalizaremos sobre la preponderancia de la escala global y su nivel internacional relacionado en el discurso social dirigido a la PA (di Pasquo et al., 2011).
} 
(I) El discurso ambiental, que estará representado aquí por Edward Goldsmith, líder ambientalista y uno de los fundadores de la revista "The Ecologist" (1969). Además Goldsmith participó en la redacción del "Manifiesto para la supervivencia" (1971), que sirvió de base para el nacimiento del Partido Verde británico (Calabró, 1992). El discurso ambiental ha demostrado ser un discurso de vital importancia en la coyuntura dada por la PA. No sólo porque fueron los ambientalistas los primeros en advertir y denunciar la crisis ecológica, sino porque además han dado visibilidad a voces locales (Bowler, 1998).

(II.1 y II.2) El discurso de disciplinas o áreas del conocimiento, en torno al cual nos detendremos, por un lado, en el discurso de la economía, representado por la Organización para la Cooperación y el Desarrollo Económico (OCDE), establecida en 1961. El objetivo principal de esta organización se encuentra dirigido a que los países miembro (que son 30) logren los niveles más elevados de crecimiento económico sostenible. La importancia de este actor social (la OCDE) descansa en el alcance y el modo de vincular a los Estados nación mediante los acuerdos internacionales que se aplican en diferentes regiones del planeta (Gilpin, 2006; Galván Martínez et al., 2016).

Por otro lado, en este apartado se considerará el discurso de la ecología, una de las ramas de la biología. Dicha disciplina dirige sus estudios a la interacción entre las especies y la de éstas con su ambiente (Burel y Baudry, 2004; Odum y Barrett, 2006). La recuperación del discurso disciplinar (sea ecológico o económico) encuentra su justificación en la legitimidad que aportan al resto de los discursos, al representar las "voces autorizadas" de la coyuntura ambiental (di Pasquo et al., 2018; Lebreton e Imbernon, 2017). Y es que tanto lo ecológico como lo económico se han vuelto casi "vías obligadas" dentro de la gestión ambiental (Odum y Barrett, 2006).

(III) El discurso de las declaraciones ambientales. En particular repararemos en "La Declaración de Río sobre el Medio Ambiente y el Desarrollo" realizada en 1992. El fragmento citado corresponde al principio núm. 12 de dicha declaración. Pero también nos detendremos en "La declaración de Río Alternativa" (1992); declaración que recibió el apoyo del Foro de las ONG Brasileñas, la Red del Tercer Mundo, la Alianza de los Pueblos del Norte, el Pacto de Acción Ecológica Latina y ENDA-Tercer Mundo. Cabe destacar que las Declaraciones Ambientales, y sobre todo las de Naciones Unidas, han cobrado gran relevancia en el contexto de la PA, funcionando "como un norte" o "como una guía de sugerencias" (aunque sin ningún valor legal). A su vez, son numerosos los Estados nación y las empresas multinacionales que se han adherido a estas declaraciones (Grinberg, 1999).

(IV) El discurso de la literatura ambiental, ejemplificado aquí por Eduardo Galeano, periodista y escritor uruguayo. El fragmento presentado corresponde a una selección de "textos verdes" de su libro titulado Úselo y tírelo. El mundo visto desde una ecología latinoamericana. La relevan- 
cia de introducir el discurso de la literatura ambiental descansa, en buena medida, en el público al que se encuentra dirigido. Teniendo la particular función de introducir a mujeres y hombres "de a pie” en el tema aquí considerado, la PA (Sáez, 2005).

(V) El discurso de las organizaciones no gubernamentales, aquí nos detuvimos en el "Instituto Worldwatch" fundado en 1974 por Lester Brown. Esta ONG publica un informe anual denominado, "The State of The World" (El estado del mundo). Dicho informe (publicado en 12 idiomas), ha funcionado como una "guía" para varios gobiernos, empresas privadas y también, para la acción ciudadana. Uno de los aspecto que hace interesante a este actor social es que, desde sus inicios, fue presentado como un centro de investigación dedicado a estudiar los problemas ambientales de alcance global (Brown, 1994). Justamente se presenta como una institución independiente que desarrolla su trabajo en una escala planetaria; lo que aquí hemos denominado con la marca global de la PA.

(VI) El discurso de programas internacionales, que estará representado aquí por el Panel Intergubernamental de Expertos sobre el Cambio Climático (IPCC) creado en 1988. Dicho programa tiene el objetivo de analizar e interpretar información científica, técnica y socioeconómica para poder comprender el fenómeno del cambio climático. Se presentan dos de las cinco conclusiones más importantes del IPCC, aparecidas en uno de los informes técnicos del año 1992. Los programas internacionales han cobrado una enorme relevancia en la coyuntura ambiental, involucrando científicos de diferentes áreas (ecología, ciencias de la tierra, agronomía, entre otras) y la adhesión de diversos Estados nación. Es a partir de estos programas que la comunidad científica no sólo ha encontrado un lugar desde dónde hablar, sino que además, han sido utilizados para generar diagnósticos y proyecciones de la situación ambiental a nivel global (di Pasquo et al., 2018).

(VII) El discurso de la publicidad "verde”, ejemplificado por una publicidad lanzada en 1991 por la empresa automotriz Mercedes-Benz. La relevancia de destacar el discurso publicitario se relaciona con la trascendencia de la PA en el escenario público. Fue con la "llegada" de este tema al público en general, que se registró en los estudios de consumo, marketing y publicidad la proliferación de nociones como "green marketing", "green advertising" o "green communication" (Delgado, 2002). A este respecto, la publicidad verde no sólo ha servido como una estrategia de venta, sino que además ha aportado ciertos significados en torno a la PA.

Recordemos que la tipología presentada descansa en la idea de que cada uno de estos discursos supone la intervención de diferentes condiciones de producción, es decir, de al menos un ámbito o sector social asociado al discurso. Estos ámbitos cumplen una función legitimadora, es decir, certifican dichos discursos en el contexto de la PA (Dreyfus y Rabinow, 1988; Foucault, 2010; di Pasquo, 2013). 
Antes de avanzar con la presentación de la Tabla 1, es conveniente realizar algunas advertencias: i) Se trata de una aproximación cualitativa (y no cuantitativa) es decir, se pretende representar los tipos discursivos que han adquirido cierto reconocimiento en el contexto de la PA. ii) Muchos de los discursos aquí señalados han sido superados o bien, fuertemente criticados, y salvo por Galeano (discurso de literatura ambiental), Goldsmith (discurso ambiental), y "La declaración de Río Alternativa" el resto de los discursos mencionados representan, en buena medida, los intereses de empresas multinacionales y de los mal llamados países del primer mundo. Por lo tanto, nuestro interés por presentar estos discursos no se vincula con su "línea ideológica" sino más bien, por pertenecer éstos al período histórico estudiado. iii) Los fragmentos citados deben ser tratados únicamente como ejemplares de los tipos discursivos aquí considerados. Es decir, no se analiza cada discurso por separado, sino que el enfoque elegido se dirige a estudiar el conjunto de los discursos, intentando precisar sus "puntos de contacto".

La Tabla 1 presenta en la primera columna los tipos de discurso; en la segunda, citas que sirven de ejemplo del énfasis que se pone en la marca global (el invariante discursivo en el contexto de una frase de longitud variable) y finalmente, en la última columna se indica al menos un ámbito o sector social vinculado con la producción del tipo discursivo.

\section{Tabla 1. Tipo de discurso, fragmento-marca global y condiciones de producción}

\section{Tipo de Discurso}

I. Ambiental

西, 1 Disciplinar:

II. 1 Disciplinar:

Economía

\section{Fragmento y marca global}

"Si nuestra sociedad industrial es destructiva, en lugar de beneficiosa, es porque se la conduce hacia la expansión continua, es decir, hacia el desarrollo económico que implica la sustitución de la biósfera, o sea el mundo de los seres vivos, que es el mundo auténtico, por una organización totalmente diferente de las cosas, a la que podríamos denominar tecnósfera, o sea el mundo de los artefactos humanos, que es el mundo suplantador" (Goldsmith, 1991: 44).

"Recomendaciones del consejo para la aplicación de instrumentos económicos en las políticas ambientales (OCDE, enero de 1991). Entre otras cosas, los países miembros deberán: Esforzarse por lograr más acuerdos internacionales sobre el uso de instrumentos de políticas ambientales para resolver problemas ambientales regionales y globales, además de asegurar el desarrollo sostenible" (Reproducida en Gilpin, 2006: 142)
Condiciones de producción

Sectores de protesta

Estados nación y área del conocimiento 


\begin{tabular}{|c|c|c|}
\hline $\begin{array}{l}\text { II.2 Disciplinar: } \\
\text { Ecología }\end{array}$ & $\begin{array}{l}\text { "Los fenómenos a nivel de paisaje también están recibiendo cada vez más } \\
\text { atención en cuanto las cuestiones del cambio global se hacen más prominen- } \\
\text { tes. Por lo tanto, los métodos para analizar e interpretar la heterogeneidad } \\
\text { a escalas espaciales grandes son cada vez más importantes para los es- } \\
\text { tudios ecológicos" (Turner, 1990: 21)." }\end{array}$ & $\begin{array}{l}\text { Universidades } \\
\text { y área del } \\
\text { conocimiento }\end{array}$ \\
\hline $\begin{array}{l}\text { III. La Declaración } \\
\text { de Río... }\end{array}$ & $\begin{array}{l}\text { "Las medidas destinadas a tratar los problemas ambientales transfron- } \\
\text { terizos o mundiales deberían, en la medida de lo posible, basarse en un } \\
\text { consenso internacional" (Reproducida en Grinberg, 1999: 199) }\end{array}$ & $\begin{array}{l}\text { Estados nación, } \\
\text { ONG y empresas } \\
\text { multinacionales }\end{array}$ \\
\hline $\begin{array}{l}\text { Declaración de Río } \\
\text { "Alternativa" }\end{array}$ & $\begin{array}{l}\text { "Tenemos conciencia de la contradicción existente entre el modelo de civili- } \\
\text { zación dominante, injusto e insostenible, construido sobre el mito del creci- } \\
\text { miento ilimitado y que ignora los límites finitos de la Tierra. Entende- } \\
\text { mos, por eso, que la salvación del planeta y de sus pueblos presentes y } \\
\text { futuros exige la creación de una nueva civilización" (Reproducida en Grin- } \\
\text { berg, 1999: 202) }\end{array}$ & $\begin{array}{l}\text { Sectores de } \\
\text { protesta }\end{array}$ \\
\hline $\begin{array}{l}\text { IV. Literatura } \\
\text { ambiental }\end{array}$ & $\begin{array}{l}\text { "Se podrá decir cualquier cosa de Al Capone, pero él era un caballero: el } \\
\text { bueno de Al siempre enviaba flores a los velorios de sus víctimas. Las em- } \\
\text { presas gigantes de la industria química, la industria petrolera y la indus- } \\
\text { tria automovilística han pagado buena parte de los gastos de la Eco-92, la } \\
\text { conferencia internacional que en Río de Janeiro se ocupó de la agonía del } \\
\text { planeta. Y esa conferencia, llamada Cumbre de la Tierra, no condenó a } \\
\text { las empresas transnacionales que producen contaminación y viven de ella" } \\
\text { (Galeano, 1994: 16) }\end{array}$ & Grupos editoriales \\
\hline V. ONG & $\begin{array}{l}\text { "Un mundo sustentable se propone brindar una visión de la sociedad am- } \\
\text { bientalmente sustentable que hoy está a nuestro alcance [...] Trabajando } \\
\text { desde una perspectiva global e interdisciplinaria, Worldwatch se espe- } \\
\text { cializa en identificar las tendencias que escaparían a la atención de quienes } \\
\text { concentran su tarea en una región geográfica en particular o trabajan en } \\
\text { una disciplina especializada" (Brown, 1994: 13-14). }\end{array}$ & ONG \\
\hline $\begin{array}{l}\text { VI. Programas } \\
\text { Internacionales: } \\
\text { IPCC }\end{array}$ & $\begin{array}{l}\text { "[Conclusión 1] La temperatura media en la superficie terrestre ha au- } \\
\text { mentado en los últimos } 100 \text { años entre } 0.3 \text { y } 0.6^{\circ} \mathrm{C} \text {. } \\
\text { [Conclusión 2] Con los datos obtenidos de los modelos de simulación y si se } \\
\text { verifica la duplicación de las concentraciones actuales de CO2, previsible- } \\
\text { mente la temperatura media terrestre aumentará entre } 1.5 \text { y } 4.5^{\circ} \mathrm{C} \text { " } \\
\text { (Reproducidas en Azcárate y Mingorance, } 1997: 43-44)\end{array}$ & $\begin{array}{l}\text { Estados nación } \\
\text { y asociaciones } \\
\text { científicas }\end{array}$ \\
\hline $\begin{array}{l}\text { VII. Publicidad } \\
\text { "verde" }\end{array}$ & $\begin{array}{l}\text { "Para esta Estrella no hay recambio. No tenemos un compromiso solo con } \\
\text { la estrella de nuestro logotipo. También lo tenemos con la estrella en la que } \\
\text { vivimos: nuestro planeta. Y ese compromiso lo tomamos tan en serio como } \\
\text { el primero" (Reproducido en Delgado, 2002: 246) }\end{array}$ & $\begin{array}{l}\text { Empresas } \\
\text { multinacionales }\end{array}$ \\
\hline
\end{tabular}

Fuente: elaboración propia

" Cita original: "Landscape-level phenomena are also receiving increasing attention as questions of global change become more prominent. Therefore, methods to analyze and interpret heterogeneity at broad spatial scales are becoming increasingly important for ecological studies" (Turner, 1990: 21). 
Por medio de los diferentes discursos presentados, podemos sugerir que en ciertos enunciados de longitud variable, se reconoció la presencia de aquella marca global indicado para el tema de la PA. Asimismo, de la comparación entre estos discursos (todos ellos dirigidos al tema de la PA) se puede admitir también que dicha marca (o bien sus variaciones) puede ser tratada como un invariante discursivo. Dicho con otras palabras, la comparación entre distintos discursos (los cuales suponen diferentes condiciones de producción), permite "trazar una diagonal" que "interconecta" a los discursos analizados en-lo-global de la PA. Ahora bien, este ejercicio comparativo tuvo por objeto, restituir buena parte del discurso social dirigido a este tema:

El solo hecho de hablar del discurso social en singular (y no evocar simplemente el conjunto contingente de los discursos sociales) implica que [...] el investigador puede identificar las dominancias interdiscursivas, las maneras de conocer y de significar lo conocido que son lo propio de una sociedad, y que regulan y trascienden la división de los discursos sociales (Angenot, 2010: 28).

Siguiendo el planteo de Angenot, podemos sugerir dos aspectos relacionados y de interés para los fines de nuestra propuesta. El primero se vincula con destacar cómo el tema de la PA ha estado determinado por la marca global. O lo que es lo mismo, la marca global ha sido un modo de significar el tema de la PA. El segundo aspecto se dirige a reconocer cierta dominancia interdiscursiva, cierta convergencia entre "todo" lo dicho, es decir una dominancia que "atravesó" la división de los discursos sociales (sean estos discursos científicos, publicitarios, literarios, de sectores de protesta, de programas internacionales, de organismos no gubernamentales o bien, el de las declaraciones ambientales). Convergencia que en el apartado próximo nos permitirá reflexionar sobre cierto "hecho" hegemónico en el contexto ofrecido por la PA.

\section{Discusión: hegemonía de lo global y orden mundial}

En el apartado anterior hemos indicado cómo cierta dimensión espacial de orden global se ha convertido en una "fórmula exitosa" y recurrente entre los discursos que tienen como tema la PA. A partir de este punto del trabajo resulta interesante interrogarse sobre la "saturación" que supone dicha fórmula en el seno del discurso social estudiado. Para poder elaborar dicho interrogante, nos parece fundamental introducir una breve caracterización de lo que entendemos por hegemonía o "hecho" hegemónico al nivel de los discursos:

Hegemonía - La noción de hegemonía que aquí consideramos debe ser comprendida en términos productivos más que prohibitivos. A este respecto se debe reconocer en la noción de he- 
gemonía cierto poder que incita, que suscita, o bien, que produce (Deleuze, 2008). En esta línea argumental, la producción a la que "invita" un "hecho" hegemónico, será la de obligar a decir: "la censura no es sólo prohibición, sino sobre todo compulsión, imposición de hablar según la doxa: 'la lengua es, esencialmente fascista; porque el fascismo, no es impedir decir, es obligar a decir" (Angenot, 1998: 32). Acorde con lo mencionado, la noción de hegemonía presentada opera en el nivel de los discursos o bien, a través de ellos. De esta manera "la hegemonía establece los límites de lo decible y de lo pensable en unas coordenadas sociohistóricas" (Dalmasso y Fatala, 2010: 10). Conviene enfatizar una vez más esta última idea, ya que mediante la aparente diversidad del discurso social se establece una hegemonía que satura todo el espacio de lo pensable y de lo decible; hegemonía que se convierte en "el médium obligado de todo pensamiento, de toda expresión, aún paradojal, de toda comunicación” (Angenot, 1998: 22).

Teniendo presente esta caracterización, resulta oportuno interrogarse sobre cuál es el modo en que puede ser abordado un "hecho" hegemónico al nivel de los discursos. Una manera de abordar el "hecho" hegemónico se vincula con la "detección" de cierta temática que se encuentra instalada. Es decir, se trata de reconocer un tema que se ha establecido o bien se ha "naturalizado" en el seno del discurso social. De este modo, dicho tema se presentará como un denominador común para los distintos discursos, o lo que es igual, se presentará como un "paso obligado" para los diferentes discursos que componen el discurso social. A su vez, se agrega también que dicha temática vendrá acompañada de aspectos que se encuentren parcialmente construidos o prefabricados. En relación con lo antes dicho, Angenot (2010: 43) menciona: "La hegemonía se presenta entonces como una temática, con [...] 'problemas' parcialmente preconstruidos, intereses ligados a objetos cuya existencia y consistencia no parecen ofrecer dudas, ya que el mundo entero habla de ellos". Bajo esta perspectiva, la hegemonía se impone (o "atraviesa") la división de los discursos sociales pautando o bien regulando el campo de los debates posibles en torno a la temática instalada: "La hegemonía se compone de un repertorio de temas que se imponen a todas las mentes, de tal manera que su tratamiento abre el campo de debates y disensos normados" (Angenot, 1998: 30).

Así, al volver sobre nuestro objetivo y retomando las dos últimas citas, estamos en condiciones de ofrecer una primera conclusión parcial: el tema de la PA con su fórmula global asociada, debe ser entendido como un "hecho" hegemónico al nivel del discurso social estudiado. O lo que es lo mismo, la hegemonía se presenta en cuanto el tema prefabricado _PA global_ "satura" todos los discursos analizados. Repitamos una vez más esta última idea, que es central para los fines de nuestra propuesta: la dominancia interdiscursiva presentada en el apartado anterior — donde pudimos rastrear enunciados de longitud variable que sostienen una globalidad de la PA- nos permite sugerir la presencia de un "hecho" hegemónico. Asimismo, vale la pena advertir que para que un tema determinado pueda ser considerado hegemónico "no tiene necesidad de enunciarse 
todo el tiempo y en todo lugar. Basta con que un cierto número de enunciados sean los únicos legitimados en el nivel de la evidencia, 'indiscutibles' e insoslayables" (Angenot, 1998: 203). En este sentido, hemos destacado que los discursos analizados "descansan" sobre ámbitos y sectores sociales que vienen a conferir autenticidad o legitimidad a los mismos. Es decir, aseguradas ciertas condiciones de producción estos discursos no tienen necesidad de ser repetidos en todo momento y en todo lugar, en tanto y en cuanto son presentados como evidentes e indudables frente al resto de los discursos posibles.

\section{Administrar la Tierra}

En este apartado, argumentaremos en favor de que el "hecho" hegemónico al nivel del discurso recién presentado (la PA con su fórmula global asociada), funcionó como condición de posibilidad para la propuesta de un orden emergente de alcance mundial. O dicho de otro modo, la hegemonía discursiva ya mencionada encuentra un correlato "casi directo", con nuevas estrategias de administración que se "expresarían" al nivel del globo. De aquí que la PA no debe ser "vista" como un tema inerte o inocuo, sino muy por el contrario, debe ser entendida como una temática que tuvo (y tiene) una fuerte instrumentalidad (Foucault, 2008b). Es decir, el tema de la PA se presentará como un instrumento de justificación para sugerir una nueva administración global. Para adentrarnos en esta administración global se consideran dos autores —Lynton Keith Caldwell y Guy Béney - que bien pueden quedar inscriptos en lo que Alfie (2013: 92) denomina la escuela del ambientalismo global:

...el ambientalismo global estudia las presiones externas o supranacionales sobre el Estado-nación en asuntos ambientales. Los analistas de esta escuela marcan la importancia de los problemas ambientales globales y de la internalización de las problemáticas de este tipo. Presionan a los Estados nacionales ante el fracaso de sus políticas y las fuertes divisiones sociales por ellas generadas; los responsabilizan de crear la sociedad de riesgo global. Esta incapacidad nacional frente al deterioro del entorno da lugar a pensar en formas globales de gobernanza ambiental.

Es en el marco de este ambientalismo global, donde se reconoce que muchos de los problemas ambientales son transfronterizos y por lo tanto se da lugar a la posibilidad de una gestión de alcance global. De este modo, en nombre de la PA global y de toda la especie humana involucrada, se vela por la gestión del planeta. En Caldwell (1993: 140), encontramos un buen ejemplo de esta perspectiva: 
El crecimiento de bancos internacionales, de sociedades multinacionales, de sistemas globales de comunicación, de programas globales y regionales de investigación científica, de líneas aéreas internacionales y de esfuerzos de ayuda internacional ha creado una red tecno-económica de empresa humana capaz de extenderse por todas las partes de los mundos naturales y políticos y de someter al planeta a la influencia de un orden mundial tecno-científico-económico.

En esta primera cita, el autor destacaba que ya están dadas las condiciones para poder someter al planeta a un orden mundial; condiciones que vienen dadas por distintas instituciones y estamentos sociales de carácter internacional. A su vez, el orden mundial propuesto supondría la participación de la empresa técnica, la empresa científica y la empresa económica. Resulta pertinente advertir que los tres ámbitos sugeridos por Caldwell han sido característicos del dominio occidental vigente. Ahora bien, dentro de este planteo más general el autor le da un "lugar" fundamental al tema de la PA: "En breve, el mundo tecno-científico-económico va a cambiar ciertos detalles de las fronteras nacionales, y este desarrollo es importante para la aspiración de un orden mundial de protección medioambiental" (Caldwell, 1993: 142).

Aquí, el "rol" asignado al tema de la PA resulta crucial para los fines de nuestro trabajo, en tanto y en cuanto es el establecimiento de un orden mundial el que resultaría imprescindible para dar lugar a la protección y solución de dicha problemática. En última instancia, la PA global viene a justificar la implementación de un orden planetario. En esta línea argumental, este autor agrega que el mundo "no está y puede que no esté nunca preparado para un gobierno de ámbito mundial, pero no puede resolver el objetivo a nivel planetario de la humanidad a través del orden existente de estados nacionales soberanos" (Caldwell, 1993: 166). Siguiendo la última cita, por un lado se afirma que el orden actual distribuido en Estados nación resulta ineficaz para abordar problemas de corte global, ${ }^{2}$ mientras que por otro lado se sugiere un gobierno de orden mundial; haciéndose la salvedad de que en algún momento del futuro estaremos lo suficientemente preparados para ello. De esta manera, en tanto estamos en presencia de problemas ambientales globales, no sólo se justificaría un orden mundial tecno-científico-económico, sino también según Caldwell, un gobierno para la Tierra.

Sobre la propuesta de orden mundial, vale la pena detenerse en la perspectiva crítica que ofrece Guy Béney. Este autor menciona al respecto: "Se trata de [...] perfeccionar modelos de simulación del medio ambiente global, instalar sistemas de vigilancia continua (redes de satélites, etc.), que deben constituir un observatorio internacional. Se instala un verdadero control del planeta, con miras a 'administrar la Tierra”' (Béney, 1996: 187). Y más adelante agrega:

\footnotetext{
${ }^{2}$ Sobre la insuficiencia de los Estados nación para abordar macropeligros, véase también la aproximación de Ulrich Beck en Pérez (1999: 235).
} 
Esta "geocracia" en ascenso que conjunta saber y poder, es juez y parte a la vez, de dominio masculino y racionalizante, occidental más que internacional, corre el riesgo de atentar en el mundo entero contra la libre opción de las poblaciones y de sus representantes (Béney, 1996: 188).

En relación con estas dos últimas citas, se puede destacar entonces que la aspiración a un orden mundial (como el que propone Caldwell), viene fuertemente asociado con cierta aspiración por controlar el planeta mediante la implementación de todo un dispositivo de "observación occidental". Y esta "geocracia" tecno-científico-económico emergente, vendría a atentar como mínimo contra las opciones de los Estados nación. Por último, Béney (1996: 189) agrega que:

Existe el riesgo de que se imponga muy rápidamente una especie de "geodinámica humana", en la cual el conjunto de los hombres, del Norte como del Sur, simplemente para sobrevivir [...] deberán justificar ecológicamente su presencia en la Tierra como consumidores obligados [...] ¿Qué será entonces de las masas de "consumidores de medio ambiente" [...] que no encuentran modo de integrarse en este nuevo reparto de cartas, y juzgados por esto no solamente inútiles sino nocivos para la salvaguarda de todos?

Siguiendo la argumentación de este autor, se corre el riesgo de que todos los Estados nación (y cada hombre sobre la superficie terrestre) independientemente de sus diferencias, deban justificarse ecológicamente frente a un orden mundial determinado.

Recuperadas las posiciones y algunos de los "elementos" ofrecidos por estos dos autores (Caldwell y Béney) se hace imprescindible volver sobre la idea central de este apartado. Básicamente, sugerimos que el "hecho" hegemónico al nivel del discurso (apartado 3), "corrió de un modo paralelo" a la pretensión de un orden de alcance mundial. Ahora bien, esta última afirmación merece una mayor profundización. En rigor, la PA de características globales, es la que viene a justificar, según Caldwell, un orden de vigilancia y de control mundial. Dicho de otra manera, la sugerencia de un orden a nivel planetario descansó en buena medida sobre la idea de que se presentan problemas ambientales que afectan a todas las naciones y a todos los seres humanos del planeta Tierra. Y si a esto último le agregamos que "no hay pensamiento que preexista al lenguaje y que se exprese (bien o mal) a través suyo" (Verón, 1987: 70) esta idea o pensamiento rastreado en las citas de Caldwell — de que se presentan problemas ambientales globales — encuentra una relación inmediata con la hegemonía presentada al nivel del discurso. Así, aquella hegemonía discursiva dada mediante el tema "naturalizado" de una PA global viene a funcionar como instrumento y condición de posibilidad para la propuesta de una "geocracia" (Béney). 
Resta decir que nuestra posición de ninguna manera invalida que frente a una PA dada, que supone la intervención de más de una nación, no deban presentarse soluciones que las implique. Como sugiere Jesús Guzmán Pineda (1993) "estos problemas deben ser resueltos con el esfuerzo de la mayoría de los países" (252). Ahora bien, las soluciones no pueden simplificarse bajo medidas valorativas universales, en nombre de un orden mundial de carácter occidental. En todo caso, dichas soluciones debieran considerar las diferencias constitutivas de las distintas naciones involucradas que por otro lado, las hay de todo tipo: valorativas, económicas, políticas, culturales, por nombrar sólo las más evidentes. Pero a la vez, tampoco se pueden evadir las soluciones posibles a un problema ambiental determinado apelando a "diferencias insalvables" entre las naciones implicadas. En efecto, la radicalización por el respeto de las diferencias puede derivar en actitudes conservadoras que suelen ser usadas para justificar las fuertes desigualdades que se presentan entre los países, tal como las que se reconocen entre los países del Norte y el Sur (De Sousa Santos y Rodríguez Garavito, 2007; Alfie, 2002). Así, reconocer la relevancia de nuestras diferencias culturales no debe llevarnos a olvidar nuestra "naturaleza ecológica" (Riechmann, 1998).

En este punto es imprescindible señalar dos posiciones nocivas y extremas que demarcan las discusiones dirigidas a la tensión global-local. Por un lado, se puede "caer" en lo que podríamos denominar como universalismo-global, el cual se caracteriza por establecer un conjunto de condiciones ecológicas "objetivas" y "deseables" para toda la humanidad. Y en nombre de estas "buenas intenciones ambientales" se justifica la opresión de las diferencias (aquí se puede aproximar la posición de Caldwell). Por otro lado, nos encontramos con otra posición igual de nociva, que podríamos denominar relativismo-local. En el cual, las prácticas y valoraciones ambientales locales no deben corromperse frente a las prácticas y valoraciones ambientales "externas" (aquí se puede aproximar la posición de Béney). Pero como mencionamos, el relativismo tiene como problema que fácilmente puede ligarse con una actitud conservadora, donde las injusticias ambientales se respondan con una "tolerancia hacia las diferencias". Ahora bien, dicha tolerancia dista de ser neutral y en todo caso, termina por representar una adhesión al status quo. Por ello, resulta imprescindible evitar tanto un relativismo-conservador como un universalismo-opresor; como afirma Jorge Riechmann (1998: 9): "En definitiva, la opresión política y social puede justificarse tanto apelando al relativismo ético como al universalismo moral, y de ello hay ejemplos sobrados en la historia lejana y en la reciente".

\section{Administrar la Tierra y glocalización}

Es importante destacar que la hegemonía de lo global (señalada en el apartado 3) no debe ser entendida en términos totalizantes o monolíticos. Es decir, la presencia del "hecho" hegemónico en nada impide que se reconozcan contradiscursos o bien, discursos que operen en direcciones dife- 
rentes al discurso hegemónico. Un buen ejemplo de discurso disidente que viene a problematizar la idea de una PA global, estuvo dada por las discusiones dirigidas en torno al concepto de glocalización. Aunque su acepción es más antigua, para fines del periodo histórico elegido (1989-1995) Roland Robertson (1995) recupera dicha noción. Este autor y otros como Ulrich Beck, destacan mediante este concepto que el proceso de la globalización es acompañado sistemáticamente por un proceso de localización.

De modo general, la tesis presentada por estos autores complejiza la simplificación de que el proceso de la globalización deriva en una convergencia creciente de los contenidos culturales (sean estos políticos, económicos, tecnológicos o bien, ambientales). De este modo, se afirma que: "La globalización —aparentemente lo muy grande, lo exterior, lo que sobreviene al final y sofoca todo lo demás-, es asible en lo pequeño y lo concreto, in situ, en la propia vida" (Beck, 2008: 80). Siguiendo esta línea argumental, no alcanza con afirmar que la PA es de orden global — tal como se ha destacado desde el discurso hegemónico- sino que además se debe agregar que dicha problemática, aun cuando afecte a toda la especie humana, termina por ser internalizada, absorbida y comprendida de modos diferentes en lo local.

Bajo esta perspectiva de la glocalización, queda problematizada entonces la idea de que un gobierno de alcance mundial (como el sugerido por Caldwell) atente contra las diferencias culturales que se dan entre los Estados nación. Dichas diferencias no se borrarían en nombre de "bondades universales", las cuales en última instancia serían absorbidas y comprendidas de distintas formas. Sin embargo, no debe perderse de vista que aun cuando grupos locales interpreten de manera diferenciada ciertos lineamientos ambientales "universales" — dictados por un orden global—, en nada impide que se establezcan relaciones asimétricas. Como sugiere Zygmunt Bauman (2013: 162): "Las cuasi soberanías, divisiones territoriales y segregaciones de identidad que impone y promueve la globalización de los mercados y de la información no reflejan la diversidad de socios en pie de igualdad" (Bauman, 2013: 94). En efecto "si los encargados de un orden local se vuelven demasiado entrometidos y molestos, siempre se puede apelar a las leyes globales para cambiar los conceptos del orden y las reglas del juego locales". De aquí que en nombre de una PA global —pero ahora "leída" en clave glocal — también se puedan establecer relaciones asimétricas entre un orden mundial y grupos locales.

\section{Conclusiones}

Para finalizar resulta pertinente profundizar en algunos aspectos de nuestra argumentación. Hemos destacado que en el discurso social dirigido al tema de la PA, se reconoce cierta regulación 
de lo que se puede decir y de lo que se puede pensar. En rigor, la hegemonía discursiva propuesta operó por medio de la instalación de cierta temática que adoptó cierta forma — la global-y que rara vez fue cuestionada. O lo que es lo mismo, se implantó para el periodo indicado, cierto tema preconstruido o prefabricado que "posee" una fuerte preponderancia. En este sentido, se debe insistir en que este tema prefabricado asume una relación asimétrica entre lo global y lo local. Resulta interesante advertir que esta asimetría contrasta fuertemente con aquellas posiciones que destacan al sujeto como "pilar" fundamental en la construcción de modos de vida que sean sustentables. Como sugiere Max-Neef, la reforma de la "persona-objeto" a la "persona-sujeto" es también un problema de escala (Ortiz Paniagua y Ortega, 2016).

Así, la asimetría global/local presupuesta en el discurso social hegemónico creemos que fomenta relaciones de dependencia que van, como es de esperarse, de la escala global a la escala local. Consideramos que la generación de modos de vida sustentables no deben ser la mera expresión de un conjunto de discursos dominantes y que, en todo caso, se debe dar lugar a la diversidad de colectivos con manifestación local. Coincidimos en que un desarrollo de las necesidades humanas no debería, por definición, estructurarse desde "arriba hacia abajo". En todo caso, lo global podría asumir un rol estimulador de las sinergias locales más que fomentar relaciones de dependencia asimétricas (Max-Neef, 1998). Dicho esto, es pertinente agregar que la hegemonía aquí presentada no debe ser concebida como un "hecho" monolítico. El que sea predominante en nada impide la presencia de contradiscursos, o bien, de discursos disidentes. Tal fue el caso de ciertas discusiones académicas que se han dirigido por ejemplo en torno al concepto de glocalización; noción que se distingue de una idea de globalización que "olvidó" el rol de lo local.

Asimismo, la aproximación que hemos realizado al seno del discurso social hegemónico, permite advertir la similitud que presentaron los discursos de disciplinas científicas con el resto de los discursos sociales vinculados al tema de la PA. Tales fueron los casos de la ecología (en el campo de las ciencias naturales) y de la economía (en el campo de las ciencias humanas). Desde la perspectiva abierta por la categoría de hegemonía o "hecho" hegemónico, estas disciplinas no presentaron diferencias cualitativas respecto al resto de los discursos estudiados a pesar de que normalmente tienen "duras" restricciones tales como el uso de lenguajes fuertemente técnicos, o por el ámbito de objetos que delimita o bien, por el conjunto de métodos que considera válidos (Foucault, 2008a). En ambas disciplinas, se presentaron "herramientas" que fueron "solidarias" con una perspectiva de alcance global — sean los acuerdos internacionales sobre el uso de instrumentos de políticas ambientales en la economía o la noción de escala espacio-temporal en la ecología-. En este sentido, estos discursos han venido a legitimar, en tanto que ciencias, aquella percepción de lo global. De aquí que se pueda sugerir que los mismos no estuvieron exentos del "proceso de 
hilvanado" del discurso social en el tema de la PA. Aun frente a preceptos autoproclamados (sobre todo en las ciencias naturales) como la objetividad epistémica o la neutralidad valorativa.

Otro punto de la argumentación merece nuestra atención. La idea de que el conjunto de los discursos estudiados confluyen en determinada temática (la PA global), invita a pensar que el hecho hegemónico supone la participación de una racionalidad que guio o que "encauzó" al discurso social. Sin embargo, esto último no se sostiene. Se debe insistir en que el establecimiento del discurso hegemónico no responde a una razón que sea "anterior" a los discursos que los fundamente y los estructure. Es decir, no se trata de la presencia y persistencia de un principio organizador que "encauza" a los discursos, considerando que estos resultan simples "derivaciones" de este principio organizador. No debe buscarse detrás de los discursos una razón homogénea, uniforme en el tiempo y en el espacio y asociada con una mínima información capaz de formar todo discurso posible. Muy por el contrario, cada discurso responde a una historia particular, con intereses, especificidades y exigencias que le son propias y que resultan ajenas al resto de los discursos.

En esta línea argumental, el discurso de la literatura ambiental, representado por Galeano, permite volver sobre estas consideraciones. En efecto, se puede advertir que en el desarrollo de su crítica este escritor simplemente "arrastra por contagio" la marca global al seno de su ensayo. De aquí que resultaría un difícil ejercicio mental argumentar — desde una perspectiva histórica— en favor de que una misma razón (en nuestro caso dirigida a la propuesta de un orden mundial) operaría tanto sobre el discurso de la literatura ambiental como sobre el discurso de la economía, por mencionar otro de los ejemplos aquí tratados. Por lo tanto, el establecimiento de la hegemonía al nivel de los discursos debe ser entendido como un proceso histórico que como tal, cae por fuera de cualquier horizonte de racionalidad imaginable. Con todo, esto en nada impide que establecido el "hecho" hegemónico emerjan voces que hacen uso de esta dominancia interdiscursiva. Hemos mencionado a modo de ejemplo algunos pasajes de la propuesta dada por Caldwell, quien valiéndose de una PA de orden global, alude a la necesidad de un gobierno de alcance mundial.

En rigor, si consideramos al poder como el nombre que se le da a una situación estratégica de dominancia (Foucault, 2008b)' podemos advertir que la estrategia de establecer un gobierno de alcance mundial puede ser entendida como una nueva forma de establecer determinadas relaciones de fuerza en detrimento de otras. Insistimos entonces en la instrumentalidad que tiene el tema de la PA: en un momento donde la "moda verde" se nos impone mediante cálidas valoraciones, no conviene perder de vista que dicho tema puede resultar funcional a diferentes estrategias de dominación. Finalmente, no debemos olvidarnos que mientras problematizamos y denunciamos las relaciones de poder que se "tejen" en nombre de la PA, nos queda la enorme tarea de avanzar en soluciones que eviten tanto los universalismos opresores como también, los relativismos conservadores. 


\section{Referencias}

Alfie, Miriam (2002). "Discursos ambientales: viaje a la diversidad". Sociológica, 48, pp. 81-119.

Alfie, Miriam (2013). "Democracia deliberativa y gobernanza ambiental: ¿conceptos transversales de una nueva democracia ecológica?". Sociológica, 80, pp. 73-122.

Angenot, Marc (1998). Interdiscursividades. De hegemonías y disidencias. Argentina: Editorial Universidad Nacional de Córdoba, 219 pp.

Angenot, Marc (2010). El discurso social: Los límites históricos de lo pensable y lo decible. Buenos Aires: Siglo Veintiuno Editores, 232 pp.

Azcárate, Blanca y Mingorance, Alfredo (1997). "Modelos de desarrollo y cambio climático". Espacio, Tiempo y Forma, Serie VI, Geografía, 10, pp. 35-50.

Bauman, Zygmunt (2013). La globalización: Consecuencias humanas. México: Fondo de Cultura Económica, $174 \mathrm{pp}$.

Beck, Ulrich (2008). ¿Qué es la globalización?: falacias del globalismo, respuestas a la globalización. Buenos Aires: Paidós, 226 pp.

Béney, Guy (1996). "La ascención de los geócratas". En: Jacques Theys y Bernard Kalaora (coords.), La tierra ultrajada. Los expertos son formales. México: Fondo de Cultura Económica, pp. 186-196.

Bowler, Peter (1998). Historia fontana de las ciencias ambientales. México: Fondo de Cultura Económica, 467 pp.

Brown, Lester Russel (1994). Un mundo sustentable. Buenos Aires: Planeta, 307 pp.

Burel, Francoise y Baudry, Jacques (2004). Landscape Ecology Concepts, Methods and Applications. United States of America: Science Publishers, 362 pp.

Calabró, Fernando (coord.) (1992). Vivir ligeramente sobre la Tierra. Barcelona: Integral, 192 pp.

Caldwell, Lynton Keith (1993). Ecología. Ciencia y política medioambiental. Madrid: McGraw-Hi1l, $253 \mathrm{pp}$.

Castro, Eduardo (2011). Diccionario de Foucault. Temas, conceptos y autores. Buenos Aires: Siglo Veintiuno Editores, 433 pp.

Dalmasso, María Teresa y Fatala, Norma (2010). "Presentación”. En María Teresa Dalmasso y Norma Fatala (coords.), Marc Angenot. El discurso social: los límites históricos de lo pensable y lo decible. Buenos Aires: Siglo Veintiuno Editores, pp. 9-12.

Deleuze, Gilles (2008). Foucault. Buenos Aires: Paidós, 172 pp.

Delgado-Olivares, Fernando (2002). "Publicidad y ecología. La publicidad verde en España [19801999]" (Tesis de doctorado en Sociología). España: Universidad de Alicante, 730 pp.

De Sousa Santos, Boaventura y Rodríguez Garavito, César (2007). "El derecho, la política y lo subalterno en la globalización contrahegemónica”. En Boaventura de Sousa Santos, y César 
Rodríguez Garavito (coords.), El derecho y la globalización desde abajo: Hacia una legalidad cosmopolita. México: Anthropos, pp. 7-28.

di Pasquo, Federico; Folguera, Guillermo, y Onna, Alberto (2011). "La ecología disciplinar y la intrusión de la problemática ambiental: hacia la 'percepción de fenómenos globales"'. Observatorio Medioambiental, 14, pp. 21-39.

di Pasquo, Federico (2013). "Una historia de las condiciones de aparición de la problemática ambiental y de sus efectos sobre la matriz de la ecología disciplinar". Scientiae Studia, 11(3), pp. 557-81.

di Pasquo, Federico (2015). "La norma global y la fractura ecológica". Rev. Colombiana de Filosofía de la Ciencia, 15(30), pp. 173-195.

di Pasquo, Federico; Busan, Tomas, y Klier, Gabriela (2018). "El dispositivo Problemática ambiental”. CIENCIA ergo-sum, 25(1), pp. 1-10.

Dreyfus, Hubert y Rabinow, Paul (1988). Michel Foucault: Más allá del estructuralismo y la hermenéutica. México: Universidad Nacional Autónoma de México, 240 pp.

Foucault, Michel (2008a). El orden del discurso. Buenos Aires: Tusquets, 78 pp.

Foucault, Michel (2008b). Historia de la sexualidad 1: la voluntad de saber. Argentina: Siglo Veintiuno Editores, $154 \mathrm{pp}$.

Foucault, Michel (2010). La arqueología del saber. Buenos Aires: Siglo Veintiuno Editores, 274 pp.

Foucault, Michel (2013). "Para una política progresista no humanista. Respuesta a una pregunta”. En Eduardo Castro (coord.), ¿Qué es usted, profesor Foucault? Sobre la arqueología y su método. Buenos Aires: Siglo Veintiuno Editores, pp. 193-221.

Galeano, Eduardo (1994). Úselo y tírelo. Buenos Aires: Booket, 123 pp.

Galván Martínez, Danaé; Fermán Almada, José Luis, y Espejel, Ileana (2016). “¿Sustentabilidad comunitaria indígena? Un modelo integral”. Sociedad y Ambiente, 4(11), pp. 4-22.

Gilpin, Alan (2006). Economía ambiental. Un análisis crítico. México: Alfaomega, 334 pp.

Goldsmith, Edward (1991). "La sociedad comunitaria”. En Fernando Calabró (coord.), Vivir ligeramente sobre la Tierra. Barcelona: Integral, pp. 43-50.

Grinberg, Miguel (1999). Ecofalacias. Buenos Aires: Galerna, 224 pp.

Gudynas, Eduardo (2015). Derechos de la naturaleza. Ética biocéntrica y políticas ambientales. Argentina: Tinta Limón, 320 pp.

Guzmán Pineda, Jesús Ignacio (1993). "El papel de la sociedad en el medio ambiente o la búsqueda de una sociología ambiental". Sociológica, 23, pp. 245-262.

Latchinian, Aramis (2010). Globotomía. Caracas: Puntocero, 254 pp. 
Lebreton, Clotilde e Imbernon, Jacques (2017). “Controversia científica y democrática: el caso de la recategorización del Parque Nacional del Nevado de Toluca, México". Sociedad y Ambiente, 5(14), pp. 5-30.

Maingueneau, Dominique (1980). Introducción a los métodos de análisis del discurso. Argentina: Hahette.

Max-Neef, Manfred (1998). Desarrollo a escala humana. Conceptos, aplicaciones y algunas reflexiones. Barcelona: Icaria Editorial.

Meyer, John (2000). “Globalization: Sources and Effects on National States and Societies”. International Sociology, 15(2), pp. 233-248.

Odum, Eugene y Barrett, Gary (2006). Fundamentos de Ecología. México: Cengage Learning Editores, 600 pp.

Ortiz Paniagua, Carlos Francisco y Ortega Gómez, Priscila (2016). "Retomando fundamentos y paradigmas para el tránsito de la crisis ambiental hacia sociedades sustentables". Sociedad y Ambiente, 4(10), pp. 113-131.

Pérez, Miguel Ángel Vite (1999). "Políticas ecológicas en la Edad del Riesgo, Ulrich Beck". Sociológica, 41, pp. 231-236.

Riechmann, Jorge (1998). "Necesidades: algunas delimitaciones en las que acaso podríamos convenir". En Jorge Riechmann (coord.), Necesitar, desear, vivir. Sobre necesidades, desarrollo humano, crecimiento económico y sustentabilidad. Madrid: Los Libros de la Catarata, pp. $11-42$.

Robertson, Roland (1995). "Glocalization: Time-Space and Homogeneity-Heterogeneity". En Mike Faetherstone, Scott Lash y Roland Robertson (coords.), Global Modernities. London: Sage, pp. 25-44.

Roudometof, Victor (2005). "Transnationalism, Cosmopolitanism and Glocalization”. Current Sociology, 53(1), pp. 113-135.

Ruggiero, Adriana (2001). "Interacciones entre la biogegrafía ecológica y la macroecología: Aportes para comprender los patrones espaciales en la diversidad biológica". En Jorge Llorente Bousquets y Juan J. Morrone (coords.), Introducción a la biogeografía en Latinoamérica: Teorías, conceptos, métodos y aplicaciones. México: UNAM, pp. 81-94.

Sáez, Victoria Sotomayor (2005). "Literatura, sociedad, educación: Las adaptaciones literarias". Revista de Educación, núm. extraordinario, pp. 217-238.

Turner, Monica (1990). "Spatial and Temporal Analysis of Landscape Patterns". Landscape Eco$\log y, 4(1)$, pp. 21-30.

Verón, Eliseo (1974). “Acerca de la producción social del conocimiento: el 'estructuralismo' y la semiología en Argentina y Chile”. Lenguajes, Revista de Lingüistica y Semiología, 1(1), pp. 96-125.

Verón, Eliseo (1987). La semiosis social. Fragmentos de una teoría de la discursividad. Argentina: Gedisa, 238 pp. 
Verón, Eliseo (1996). "La palabra adversativa. Observaciones sobre la enunciación política”. En Eliseo Verón (coord.), El discurso político. Lenguajes y acontecimientos. Buenos Aires: Hachette, pp. 1-12.

Verón, Eliseo (2004). Fragmentos de un tejido. Argentina: Gedisa, 234 pp.

Recibido: 15 marzo 2018 Aceptado: 20 agosto 2018 